\title{
On periodic solutions of a certain third-order non-linear differential equation
}

\author{
by S. SÆ̨DZTWY (Kraków)
}

1. Consider the third-order differential equation

$$
x^{\prime \prime \prime}+a x^{\prime \prime}+b x^{\prime}+f(x)=p(t),
$$

where $a, b$ are positive constants and functions $f(x), p(t)$ are defined and continuous for all $x$ and $t$ and such that (1) has a solution satisfying any initial conditions. Moreover, suppose that $f(x)$ and $p(t)$ satisfy the following assumptions:

$$
\begin{gathered}
f(x) x>0 \quad \text { for } \quad x \neq 0, \\
|f(x)| \leqslant M \quad \text { for all } x, \\
F(x) \equiv \int_{0}^{x} f(u) d u \rightarrow \infty \quad \text { as } \quad|x| \rightarrow \infty, \\
|p(t)| \leqslant p, \quad|P(t)| \equiv\left|\int_{0}^{t} p(s) d s\right| \leqslant p \quad \text { for all } t .
\end{gathered}
$$

THEOREM 1. If (2), (3), (4), (5) hold and function $p(t)$ is periodic with the period $\omega$, then equation (1) has at least one periodic solution with the period $\omega$.

This theorem strengthens the result of J. O. C. Ezeilo ([1]), who has proved the existence of a solution of (1) with the period $n \omega$, where $n \geqslant 1$ is an integer. In [1] in place of condition (2) it is assumed that $f(x) x>0$ beyond the segment $\left\langle x_{1}, x_{2}\right\rangle\left(-1<x_{1} \leqslant 0 \leqslant x_{2}<1\right)$, but this is inessential. As can be proved, the replacement of the function $f(x)$ in a compact set by any continuous function has no effect upon the boundedness of the solutions. Moreover, the assumption $f(x) \operatorname{sgn} x \geqslant m>0$, $|x| \geqslant 1$ used in [1] is replaced by the more general assumption (4).

The method of proof is the same as the one used in the 3 part of [1]. It consists in showing, by the use of the Leray-Schauder technique for Banach spaces, that a certain operator in the Banach space induced by (1) has a fixed point. 
2. Proof of Theorem 1. Let us consider alongside with (1) the family ( $\left.\mathrm{E}_{\mu}^{*}\right)$ of differential equations

$$
x^{\prime \prime \prime}+\alpha(\mu) x^{\prime \prime}+\beta(\mu) x^{\prime}+\gamma(\mu) x+\mu^{2} f(x)=\mu p(t), \quad \mu \in\langle 0,1\rangle,
$$

where

$$
\alpha(\mu)=\mu a+(1-\mu) a_{1}, \quad \beta(\mu)=\mu b+(1-\mu) b_{1}, \quad \gamma(\mu)=(1-\mu)^{2} c .
$$

Numbers $a_{1}, b_{1}, c$ are certain positive constants chosen in such a way that the equation

$$
\varrho^{3}+a_{1} \varrho^{2}+b_{1} \varrho+c=0
$$

has real distinct roots, and the following inequalities are satisfied:

$$
\begin{gathered}
c<\min _{\mu} \alpha(\mu) \min _{\mu} \beta(\mu), \\
c<\min _{\mu \in\langle 0,1\rangle}\left(\beta^{2}(\mu) / \alpha(\mu)\right) .
\end{gathered}
$$

We shall always use in the sequel $a, \beta, \gamma$ instead of $\alpha(\mu), \beta(\mu), \gamma(\mu)$.

Let $X^{*}$ be a space of continuous functions with the period $\omega$ having the norm $\|x\|=\max |x(t)|$. We define in $X^{*}$ the operator $L$ by ascribing to any function $\varphi(t) \in X^{*}$ the function $\xi(t)$, which is the solution with the period $\omega$ of the differential equation

$$
x^{\prime \prime \prime}+a_{1} x^{\prime \prime}+b_{1} x^{\prime}+c x=\varphi(t) .
$$

It can easily be demonstrated, by finding the general solution of (9) and remembering the conditions imposed upon $a_{1}, b_{1}, c$, that the operator $L$ is well-defined on the whole $X^{*}$ and that $L$ is linear in $X^{*}$.

The equation $\left(\mathrm{E}_{\mu}^{*}\right)$ is equivalent to the equation

where

$$
x^{\prime \prime \prime}+a_{1} x^{\prime \prime}+b_{1} x^{\prime}+c x=\mu \Phi(\mu, x),
$$

$$
\Phi(\mu, x)=\left(a_{1}-a\right) x^{\prime \prime}+\left(b_{1}-b\right) x^{\prime}+(2-\mu) c x-\mu f(x)+p(t),
$$

and this is not periodic in $t$ unless $x$ and $p$ are themselves periodic. Thus if, in the sequel, we suppose that $p(t)$ has a period $\omega$, then it follows that any continuously differentiable function $x(t)$ with a period $\omega$ is a solution of $\left(\mathbf{E}_{\mu}^{*}\right)$ if and only if

$$
x=\mu L \Phi(\mu, x) \text {. }
$$

Let $X$ denote the linear space of twice continuously differentiable functions $x(t)$ with a period $\omega$, having the norm $\|x\|=\max \left|x^{\prime \prime}\right|+\max \left|x^{\prime}\right|+$ $+\max |x|$. A function $x \in X$ is a solution of $\left(\mathrm{E}_{\mu}^{*}\right)$ if and only if it satisfies the equation

$$
x-\mu T(\mu, x)=0,
$$


where the operator $T(\mu, x)$ is defined by

$$
T(\mu, x)=L \Phi(\mu, x), \quad(\mu, x) \in\langle 0,1\rangle \times X .
$$

(Observe that from the definition of $\Phi$ and $L$ it follows that the operator $T(\mu, x)$ is continuous in both arguments and completely continuous.)

Thus in order to prove Theorem 1 it is sufficient to show that the equation

$$
x-T(1, x)=0
$$

has at least one solution.

Suppose that an a priori bound $\|x\|<A$ is known to exist for any $x$ satisfying $\left(\varepsilon_{\mu}\right)$, where $A$ is a constant independent of $\mu$. Then from the Leray-Schauder theory it follows that the equation $\left(\varepsilon_{1}\right)$ has at least one solution $x \in X$.

We shall demonstrate that solutions of $\left(\mathbf{E}_{\mu}^{*}\right)$ are bounded with their first and second derivatives by a constant independent of $\mu$. In view of the definition of the norm in the space $X$, it follows that solutions of $\left(\varepsilon_{\mu}\right)$ are uniformly bounded with respect to $\mu$.

If we consider the differential system

$$
\begin{aligned}
& x^{\prime}=y, \\
& y^{\prime}=z, \\
& z^{\prime}=-\alpha z-\beta y-\mu^{2} f(x)-\gamma x+p(t) \mu, \quad 0 \leqslant \mu \leqslant 1
\end{aligned}
$$

corresponding to $\left(\mathrm{E}_{\mu}^{*}\right)$, then to prove the boundedness of solutions of ( $\left.\mathrm{E}_{\mu}^{*}\right)$ it is enough to prove the following theorem:

3. THEOREM 2. If (2), (3), (4), (5) hold, then there exists a constant $D$ independent of $\mu$ such that for any point $\left(x_{0}, y_{0}, z_{0}\right)$ the solution $x(t)$, $y(t), z(t)$ of $\left(\mathrm{E}_{\mu}\right)$ determined by the initial conditions

$$
x\left(t_{0}\right)=x_{0}, \quad y\left(t_{0}\right)=y_{0}, \quad z\left(t_{0}\right)=z_{0}
$$

satisfies the inequality

$$
|x(t)|+|y(t)|+|z(t)|<D,
$$

for $t \geqslant t_{0}+T$, where $T$ depends only on particular $\left(x_{0}, y_{0}, z_{0}\right)$ chosen.

The proof of the theorem consists in constructing in the space $E^{3} \times I$ $(I=\{t: t \geqslant 0\})$ a domain $\Delta$, with the boundary $\Sigma$, included in the domain $|x|+|y|+|z|<D, t \in I$ and such that:

(i) all trajectories of $\left(\mathrm{E}_{\mu}\right)$ cross $\Sigma$ only inwards,

(ii) each trajectory of $\left(E_{\mu}\right)$ beginning outside $\Delta$ reaches $\Sigma$ after a finite time, which depends only upon the initial point of the trajectory. 
$\Sigma$ consists of surfaces defined by conditions $W_{1}(x, y, z ; \mu)=C$, $W_{2}(x, y, z, t ; \mu)=K$, where $C, K$ are positive constants suitably chosen and $W_{1}, W_{2}$ are some functions stated in the sequel.

4. Lemma 1. Let the functions $a, \beta, \gamma$ be defined by (6), where the numbers $a_{1}, b_{1}, c$ satisfy (7) and let $k$ be a constant independent of $\mu$ such that

$$
1 / a<k<\beta / c \quad \text { for all } \mu \epsilon\langle 0,1\rangle
$$

(by (7) such a number can always be chosen); then there exist positive numbers $\varepsilon, \lambda_{1}, \lambda_{2}$ such that for $e=\varepsilon, l=\lambda_{1}, L=\lambda_{2}$ the quadratic forms

$$
\begin{array}{r}
\Phi(x, y, z ; \mu)=\gamma x^{2}+k\left(\beta y^{2}+z^{2}\right)+\alpha y^{2}+2 y z+2 \gamma(e x z+k x y)- \\
-l\left(\gamma x^{2}+y^{2}+z^{2}\right), \\
\Psi(x, y, z ; \mu)=e \gamma^{2} x^{2}+y^{2}(\beta-k \gamma)+z^{2}(k a-1)-\gamma e(y z-\alpha x z-\beta x y)- \\
-L\left(\gamma^{2} x^{2}+y^{2}+z^{2}\right), \quad \mu \epsilon\langle 0,1\rangle
\end{array}
$$

are positive semi-definite and this property holds uniformly in $\langle 0,1\rangle$.

Proof. We shall prove that if $\varepsilon, \lambda_{1}, \lambda_{2}$ are sufficiently small, then for $\mu \epsilon\langle 0,1), e=\varepsilon, l=\lambda_{1}, L=\lambda_{2}$ the principal minors of the matrices of the forms $\Phi$ and $\Psi$ are positive. These minors are

$$
\begin{aligned}
& M_{1}(l, e, \mu)=k-l, \\
& M_{2}(l, e, \mu)=(k \beta+a-l)(k-l)-1, \\
& M_{3}(l, e, \mu)=\gamma(1-l)(k \beta+a-l)(k-l)+2 k \gamma^{2} e-\gamma^{2} e^{2}(k \beta+a-l)- \\
& N_{1}(L, e, \mu)=k a-1-L, \\
& N_{2}(L, e, \mu)=(k a-1-L)(\beta-k \gamma-L)-\frac{1}{4} \gamma^{2} e^{2}, \\
& N_{3}(L, e, \mu)=\gamma^{2}\left[(e-L)(\beta-k \gamma-L)(k a-1-L)-1 / 4 e^{2} \alpha \beta \gamma-\right. \\
& \quad-\frac{1}{4} e^{2} a^{2}(\beta-k \gamma-L)-\frac{1}{4} e^{2} \beta^{2}(k a-1-L)-1 e^{2}(k-l), \\
&
\end{aligned}
$$

We have

$$
\begin{gathered}
M_{i}(0,0, \mu)>0, \quad N_{i}(0,0, \mu)>0 \quad(i=1,2) \quad \text { for } \quad \mu \epsilon\langle 0,1\rangle ; \\
M_{3}(0,0, \mu)=\gamma(k \alpha-1)+\gamma(\beta-\gamma k) k^{2}>0, \\
\frac{\partial}{\partial e} N_{3}(0,0, \mu)=\gamma^{2}(\beta-k \gamma)(k \alpha-1)>0
\end{gathered}
$$

by (10) for $0 \leqslant \mu<1$. In view of the continuity of functions $M_{j}, N_{j}$ $(j=1,2,3)$ the above inequalities imply that for $\varepsilon, \lambda_{1}, \lambda_{2}$ small enough the quantities $M_{j}\left(\lambda_{1}, \varepsilon, \mu\right), N_{j}\left(\lambda_{2}, \varepsilon, \mu\right)$ are positive for all $\mu$ satisfying $0 \leqslant \mu<1$. It is easy to show that $\varepsilon, \lambda_{1}, \lambda_{2}$ can be chosen independent 
of $\mu$. Thus for $e=\varepsilon, l=\lambda_{1}, L=\lambda_{2} \Phi$ and $\Psi$ are in the segment $\langle 0,1$ ) positive definite. Since $M_{\mathrm{g}}(l, e, 1)=N_{\mathrm{a}}(L, e, 1)=0$, then from the above considerations it follows immediately that $\Phi(x, y, z ; 1), \Psi(x, y, z ; 1)$ are positive semi-definite, and this ends the proof of Lemma 1.

5. Suppose that $k$ and $\varepsilon$ are defined as in section 4. We define functions $W_{1}(x, y, z ; \mu), W_{2}(x, y, z, t ; \mu)$ as follows:

$$
\begin{gathered}
2 W_{1}(x, y, z ; \mu)=2 \mu^{3} F(x)+\gamma x^{2}+k\left(\beta y^{2}+z^{2}\right)+\alpha y^{2}+2 y z+2 \gamma(\varepsilon x z+k x y), \\
W_{2}(x, y, z, t ; \mu)=z+a y+\beta x-\mu P(t) .
\end{gathered}
$$

By Lemma 1 we have the inequality $2 W_{1}-2 \mu^{2} F(x)-\lambda_{1}\left(\gamma x^{2}+y^{2}+z^{2}\right)$ $=\Phi(x, y, z ; \mu) \geqslant 0$, which yields

$$
g(x)+\lambda_{1}\left(y^{2}+z^{2}\right) \leqslant 2 W_{1}(x, y, z ; \mu),
$$

where

$$
g(x)=\min _{\mu<\langle 0,1\rangle}\left[2 \mu^{2} F(x)+\lambda_{1} \gamma x^{2}\right]=F(x) \frac{2 c \lambda_{1} x^{2}}{2 F^{2}(x)+c \lambda_{1} x^{9}} .
$$

Since $g(x)>0$, for all $x$ and $g(x) \rightarrow \infty$ as $|x| \rightarrow \infty W_{1}(x, y, z ; \mu)$ is positive definite and

$$
\lim _{(x, y, z) \rightarrow \infty} W_{1}(x, y, z ; \mu)=\infty
$$

uniformly with respect to $\mu$.

We define in $E^{3} \times I$ the sets

$$
\begin{gathered}
\Delta_{1}(\mu, C)=\left\{(x, y, z, t): t \in I, W_{1}(x, y, z ; \mu) \leqslant C\right\}, \\
\Delta_{2}(\mu, K)=\left\{(x, y, z, t): t \in I,\left|W_{2}(x, y, z, t ; \mu)\right| \leqslant K\right\}, \\
\Delta(\mu, K, C)=\Delta_{1}(\mu, C) \cap \Delta_{2}(\mu, K), \quad \Sigma(\mu, K, C)=\operatorname{Fr} \Delta(\mu, K, C), \\
E(r, q)=\left\{(x, y, z, t): t \in I,|x| \geqslant q, y^{2}+z^{2} \geqslant r^{2}\right\} .
\end{gathered}
$$

For arbitrary fixed positive constants $d$ and $s_{1}$, we choose a number $r_{0}$ such that for $\gamma^{2} x^{2}+y^{2}+z^{2} \geqslant r_{0}^{2}$

$$
-\lambda^{2}\left(\gamma^{2} x^{2}+y^{2}+z^{2}\right)+d(|\gamma x|+|y|+|z|) \leqslant-s_{1} .
$$

It can be found by straightforward calculation that there exists a constant $h_{1}\left(r_{0}\right)$ such that for sufficiently large $C$ the surface $W_{1}(x, y, z ; \mu)$ $=C$ considered for $y^{2}+z^{2} \leqslant r_{0}^{2}$ lies in the layer $\left|x-x_{c}\right| \leqslant h_{1}\left(r_{0}\right)$, where the number $x_{c} \geqslant \sqrt{2 C / c}$ is the solution of the equation $W_{1}(u, 0,0 ; \mu)=C$. From this it follows that a constant $h_{0}$ can be found such that for $C$ large the points of intersection of the surface $W_{1}(x, y, z ; \mu)=C$ and the plane

$$
z+\alpha y+\beta x=\beta\left(\sqrt{2 C / c}-h_{0}\right)+p, \quad \mu \epsilon\langle 0,1\rangle
$$


lie outside the domain $y^{2}+z^{2} \leqslant r_{0}^{2}$. Indeed, it is enough to choose $h_{0}$ so large that the points of (14) considered for $y^{2}+z^{2} \leqslant r_{0}^{2}$ will lie outside the domain $\left|x-x_{c}\right| \leqslant h_{1}\left(r_{0}\right)$.

LEMMA 2. Let us put $K(C)=\beta\left(\sqrt{2 C / c}-h_{0}\right)$, where $h_{0}$ is chosen as above. Suppose that the hypotheses of Theorem 2 are satisfied and let $q$ be a positive fixed constant; then there exists a number $C_{0}$ such that

$$
\operatorname{Fr} \Delta_{1}(\mu, C) \cap \operatorname{Fr} \Delta_{2}(\mu, K(C)) \subset E\left(r_{0}, q\right)
$$

holds for $C \geqslant C_{0}$ and all $\mu \epsilon\langle 0,1\rangle$.

Proof. The inequality

$$
|z+\alpha y+\beta x-\mu P(t)| \geqslant|z+\alpha y+\beta x-p|
$$

implies the inclusion of $\Delta_{2}(\mu, K(C))$ in the set $|z+\alpha y+\beta x-p| \leqslant K(C)$. Hence by the definition of $K(C)$ all points of the product $\operatorname{Fr} \Delta_{1}(\mu, C) \cap$ $\cap \operatorname{Fr} \Delta_{2}(\mu, K(C))$ are in the domain $y^{2}+z^{2} \geqslant r_{0}^{2}$ for $C \geqslant C^{*}$. To prove the inclusion of this product in the layer $|x| \geqslant q$, we will show that if $C$ is large, then for arbitrary fixed $t \in I$ the system of equations

$$
\begin{gathered}
W_{\mathbf{1}}(q, y, z ; \mu)=C, \\
W_{\mathbf{2}}(q, y, z, t ; \mu)=K(C)
\end{gathered}
$$

has no real roots.

Calculating $z$ from the second equation and putting it into the first we get the equation

$$
\frac{1}{2} y^{2} A_{1}-y\left(A_{2} \sqrt{C}+A_{3}\right)+B_{1} C+B_{2} \sqrt{C}+B_{3}=0,
$$

where $A_{1}=k \beta+k \alpha^{2}-a, A_{2}=\sqrt{2 / c}(k a \beta-\beta), B_{1}=k \beta^{2} / c-1$ and coefficients $B_{2}, B_{3}, A_{3}$ are bounded for all $(t, \mu) \in I \times\langle 0,1\rangle$.

The expression $A_{2}^{2}-2 A_{1} B_{1}=2 / c\left[(k \alpha-1)\left(\alpha c-\beta^{2}\right)+k \beta^{2}\left(c-k \beta^{2}\right)\right]$ in virtue of (8) is negative; thus

$$
\left(A_{2} \sqrt{C}+A_{3}\right)^{2}-2 A_{1}\left(B_{1} C+B_{2} \sqrt{C}+B_{3}\right)<0
$$

for large $C$ (say $C \geqslant C^{* * *}$ ), whence equation (16) has no real solutions for $C \geqslant C^{* *}$. For a constant $C$ satisfying $C \geqslant C_{0}=\max \left(C^{*}, C^{* *}\right)$ we get (15).

6. Proof of Theorem 2. Let us put $d=\max (k M+k p, p, \varepsilon p)$ and define the constants $r_{0}, q, C_{0}, K(C)$ as previously in section 5. For $c>c_{0}$ we define the function $V(x, y, z, t ; \mu)$ by

$$
V(x, y, z, t ; \mu)=\left\{\begin{array}{c}
W_{1}(x, y, z ; \mu) \\
\text { for } \quad(x, y, z, t) \in \operatorname{Fr} \Delta_{1}(\mu, C) \cap \Sigma(\mu, K(C), C), \\
\left(\frac{1}{\beta} W_{2}(x, y, z, t ; \mu)+h_{0}\right)^{2} c / 2 \\
\quad \text { for }(x, y, z, t) \in \operatorname{Fr} \Delta_{2}(\mu, K(C)) \cap \Sigma(\mu, K(C), C) .
\end{array}\right.
$$


Each point $(x, y, z, t)$ of $\Sigma(\mu, K(C), C)$ satisfies the equation

$$
\nabla(x, y, z, t ; \mu)=C \text {. }
$$

By (12) $V$ is positive definite for all $\mu \epsilon\langle 0,1\rangle$ and

$$
\lim _{(x, y, z) \rightarrow \infty} V(x, y, z, t ; \mu)=\infty
$$

uniformly in $(t, \mu) \epsilon I \times\langle 0,1\rangle$. By (11) the surface $\Sigma(\mu, K(C), C)$ is included in the cylindrical domain $D(C)$

$$
D(C)=\left\{(x, y, z, t): t \in I, \frac{1}{2} g(x)+\frac{1}{2} \lambda_{1}\left(y^{2}+z^{2}\right) \leqslant C\right\},
$$

whose directrix is bounded for each positive $C$.

The derivative of $V$ along any solution of $\left(\mathrm{E}_{\mu}\right)$ is negative. In fact, if $(x, y, z, t) \in \operatorname{Fr} \Delta_{2} \cap \Sigma$, then $V=W_{2}$ and by Lemma $2|x| \geqslant q$. Hence

$$
V^{\prime}=\frac{c}{\beta}\left(\frac{1}{\beta} W_{2}+h_{0}\right) W_{2}^{\prime}=-\sqrt{\frac{2 \overline{C c}}{\beta^{2}}} f(x) \operatorname{sgn} x \leqslant-s_{2}<0 .
$$

$s_{2}$ is positive because the expression $\sqrt{2 C \bar{c} / \beta^{2}}$ is positive for all $\mu \epsilon\langle 0,1\rangle$, $C \geqslant C_{0}$ and from the inequality $|x| \geqslant q$ and the boundedness of $\Sigma(\mu, K(C), C)$ it follows that $|f(x)|$ has a positive lower bound. If $(x, y, z, t) \epsilon \operatorname{Fr} \Delta_{1} \cap \Sigma$, then $V=W_{1}$ and the derivative of $V$ along the trajectory of $\left(\mathrm{E}_{\mu}\right)$ is given by the expression

$$
\begin{aligned}
V^{\prime}=W_{1}^{\prime}=-\mu^{2} \gamma \varepsilon f(x) x-\varepsilon \gamma^{2} x^{2}-y^{2}(\beta-k \gamma)-z^{2}(k a-1)+ \\
+\varepsilon \gamma(y z-a x z-\beta x y)-k z \mu^{2} f(x)+p(t)(k z+y+\varepsilon \gamma x) .
\end{aligned}
$$

By Lemma 2 from $(x, y, z, t) \in \operatorname{Fr} \Delta_{1} \cap \Sigma$ it follows that $y^{2}+z^{2} \geqslant r_{0}^{2}$ and this implies (13). Next

$$
\begin{aligned}
W_{1}^{\prime} & =-\Psi(x, y, z ; \mu)-\mu^{2} \gamma \varepsilon f(x) x-k z \mu^{2} f(x)+p(t)(k z+y+\varepsilon \gamma x) \\
& \leqslant-\Psi(x, y, z ; \mu)+d(|\gamma x|+|y|+|z|)-\lambda_{2}\left(\gamma^{2} x^{2}+y^{2}+z^{2}\right) \leqslant-s_{1}
\end{aligned}
$$

in virtue of Lemma 1 and (13). Putting $s=\min \left(s_{1}, s_{2}\right)$, we get

$$
V^{\prime} \leqslant-s<0
$$

along the solutions of $\left(\mathrm{E}_{\mu}\right)$. Notice that the constant $s$ is independent of $t_{0}$ and $\mu$. Hence the surface $\Sigma$ defined as $\Sigma=\Sigma\left(\mu, K\left(O_{0}\right), C_{0}\right)$ satisfies (ii) for each $\mu \epsilon\langle 0,1\rangle$. $\Sigma$ has property (i) because (21) remains valid also for $C=C_{0}$. There exists a constant $D>0$ such that the set $D\left(C_{0}\right)$ defined by (18) is contained in the domain $t \in I,|x|+|y|+|z|<D$, and so $\Sigma$ also lies in this domain. Thus Theorem 2 is proved completely. 
7. For $\mu=1$ Theorem 2 gives an elementary proof of the result obtained in a different, more complicated way by J. O. C. Ezeilo ([2]). Moreover, his condition $f(x) \operatorname{sgn} x \geqslant m>0$, for $|x| \geqslant 1$ is relaxed to (4).

\section{Relerences}

[1] J. 0. C. Ezeilo, On the existence of periodic solutions of a certain third-order differential equation, Proc. Cambr. Phil. Soc. 56 (1960), pp. 381-389.

[2] - On the boundedness of solutions of a oertain differential equation of the third order, Proc. Lond. Math. Soc. (3) 9 (1859), pp. 74-114.

Regu par la Rédaction le 4.9.1963 